\title{
Epidemiología de las lesiones en los estudiantes de Ciencias del Movimiento Humano de la Universidad de Costa Rica \\ Epidemiology of injuries in students of Human Movement Sciences at the University of Costa Rica \\ Willy Gálvez Aguilar, Cinthya Campos Salazar, Yamileth Chacón Araya \\ Universidad de Costa Rica
}

\begin{abstract}
Resumen. El propósito del estudio fue determinar la tasa de incidencia de lesiones y el de riesgo de lesión en el estudiantado de primer año de la Carrera Ciencias del Movimiento Humano de la Universidad de Costa Rica durante el año lectivo 2014. Es un estudio prospectivo en el que participaron 47 estudiantes, quienes voluntariamente completaron el consentimiento informado y un cuestionario de autoreporte para conocer la presencia de lesiones y hábitos de actividad física. A los participantes se les indicó que cada lunes del semestre debían reportar la presencia de alguna lesión ocurrida durante la semana anterior y que debían sacar una cita médica para un diagnóstico preciso. Se encontró que el 31.8\% de los estudiantes (80\% mujeres y 20\% hombres) ingresaron lesionados a la carrera ( $\mathrm{p}<0.001)$. Durante el año de seguimiento, 59.5\% de la población estudiantil presentó una lesión nueva. Hubo 37 lesiones nuevas, 24 (64.9\%) en mujeres y 13 (35.1\%) en hombres, siendo las de rodilla y muslo las más frecuentes. Estas lesiones ocurren principalmente en el contexto extracurricular. El riesgo de lesión para la población fue de 0.79 y la tasa de incidencia 2.00 lesiones/1000 h de exposición. Se concluye que la población estudiantil masculina y femenina de primer año de la carrera de Ciencias del Movimiento Humano de la Universidad de Costa Rica es vulnerable a la presencia de lesiones en el tren inferior, mostrando una mayor cantidad de lesiones en el segundo semestre del año. Se necesitan estudios longitudinales para conocer la evolución y atención de las lesiones a lo largo de la carrera.
\end{abstract}

Palabras clave. Lesión, deporte, estudiantes, prevención, salud

Abstract. The purpose of the study was to determine the incidence rate of injuries and the risk of injury on first-year students of the Human Movement Sciences Program from the University of Costa Rica during the school year 2014. In this prospective study, 47 students voluntarily completed an informed consent and a self-report questionnaire to determine injuries and physical activity habits. Participants were instructed that every Monday of the semester should report any injury that occurred during the previous week, and to make a doctor's appointment for an accurate diagnostic. Results indicated that $31.8 \%$ of the first-year students ( $80 \%$ women, $30 \%$ men) entered the program already injured (p $<0.001$ ). During the follow-up year, $59.5 \%$ of the student population reported a new injury. There were 37 new lesions, 24 (64.9\%) in women and 13 (35.1\%) in men, being the knee and thigh the most frequent injuries. These lesions occurred mainly in the extracurricular context. The injury risk for the population was 0.79 and the incidence rate of 2.00 injuries/1000-h of exposure. In conclusion, the first-year male and female student population of the Human Movement Sciences Program at the University Costa Rica is vulnerable to injuries in the lower body, with a greater number of injuries in the second semester of the year. Longitudinal studies are needed to determine the evolution and care of injuries throughout the program.

Keywords. Injury, sports, students, prevention, health.

\section{Inrtoducción}

Existe evidencia contundente que indica que el aumento en la cantidad de actividad física está íntimamente relacionada con la prevención de múltiples enfermedades y con el mejoramiento de la calidad de vida delapoblación que la realice(Fiuza-Luces, Garatachea, Berger, \& Lucia, 2013). Estos beneficios son independientes de la edad, el sexo, lugar de residencia y el estado socioeconómico de quienes la practiquen. Además, se reconoce que la actividad física es una estrategia de relativamente fácil acceso para la mayoría de las personas; (Steiner et al., 2004) por ello, la formación de especialistas en Ciencias del Movimiento Humano (CIMOHU), conocida también como Educación Física, ha cobrado importancia en los últimos años.

Estos profesionales en su labor profesional determinan, entre otros aspectos, la aptitud física y motriz de las personas, y son los responsables de proponer programas de ejercicio físico para el progreso y mantenimiento, primordialmente, de la salud física, con los consiguientes beneficios en otras áreas del desarrollo (e.g., salud psicológica y social). De esta forma, este grupo laboral también colabora en el mejoramiento de la salud integral de la población en general (Moncada-Jiménez, 2005a), y para llevar a cabo estas responsabilidades profesionales, quienes ingresan a la carrera de CIMOHU deben, dentro de su plan de estudios, cursar materias que requieren de esfuerzo físico, por lo que en este grupo de estudiantes se presupone un alto nivel de actividad física a través de toda su carrera.

Esta característica del plan curricular, aunque en primera instancia coloca al estudiantado en una condición de salud favorable, también provoca el inconveniente cuando éste realiza una cantidad elevada de actividad física. Por ejemplo, en una investigación epidemiológica prospectiva realizada durante un año con 128 estudiantes de primer

Fecha recepción: 24-02-16. Fecha de aceptación: 11-11-16 Cinthya Campos Salazar

cinthyacampos4@gmail.com ingreso a la carrera de Educación Física en la Universidad de Ghent en Bélgica, se encontró que realizar más de 1000 horas de actividad física o ejercicio se relaciona con la aparición de lesiones (Goossens, Verrelst, Cardon, \& De Clercq, 2014). En ese mismo estudio se determinó que las lesiones agudas en el tren inferior fueron las más frecuentes y ocurrieron en situaciones donde no había contacto con otra persona, como por ejemplo, durante algún tipo de actividad física individual. Además, se encontró que la mayoría delas lesiones ocurrían en actividades curriculares propias de la carrera (Goossens et al., 2014).

En otra investigación de tipo prospectivo con una duración de 4 años, con estudiantes de Educación Física (Twellaar, Verstappen, \& Huson, 1996), se demostró la relevancia del estudio de lesiones en los estudiantes de Educación Física. Los investigadores reportaron cifras significativas anuales de riesgo de lesión de 1.1 a 2.1 estudiante/año, con una tasa de incidencia de lesión de 1.26 a 1.77 por cada 1000 horas de actividad deportiva. Estas cifras podrían interpretarse como un detrimento crónico del actual estudiante como un futuro profesional de Educación Física, asumiendo, entre otras cosas, que esa persona mantendría altos niveles de actividad física durante toda su vida.

En este contexto, se podría suponer que una población vulnerable a lesiones deportivas es aquella que estudia la carrera de CIMOHU, la cual se expondría una mayor cantidad de tiempo a actividades físicas, no solo como parte de su formación profesional, sino también como parte de sus hábitos de salud. Se ha estimado queun profesional deEducación Física, que labora en escuelas y colegios, se expone en promedio 25 horas semanales a sus labores (i.e., actividades físicas al aire libre o bajo techo) (Meneses \& Moncada, 2004; Moncada-Jiménez, MenesesMontero, Hidalgo-Matlock, \& Granados-Chavarría, 2004); es decir, aproximadamente $1200 \mathrm{~h}$ anuales en labores de contacto con sus estudiantes. Sin embargo, estos valores podrían subestimar la cantidad real de actividad física, pues no toma en consideración la suma de todas aquellas actividades físicas extra laborales, como por ejemplo, compromisos físico-recreativos o competitivos, que muchas veces pueden significar una carga física importante a largo plazo. 
En un estudio realizado en Holanda acerca de la incidencia de lesiones en estudiantes de Educación Física (Twellaar et al., 1996), se analizaron los datos de 136 estudiantes de primer ingreso, a quienes se les dio un seguimiento de 4 años. Al final del estudio, se reportaron 525 lesiones deportivas, 183 (34.9\%) en mujeres y 342 (65.1\%) en hombres, en donde las sesiones de cursos de deporte de la carrera (i.e., curriculares) mostraron el reporte más alto de lesiones (Twellaar et al., 1996). Estos hallazgos son similares a los encontrados usando modelos matemáticos en deportistas con mayor trayectoria competitiva, quienes acumulan una mayor cantidad de horas de exposición al ejercicio, y por lo tanto una mayor posibilidad o incidencia de lesiones (Fernández Martínez, de la Cruz Márquez, Cueto Martín, Salazar Alonso, \& de la Cruz Campos, 2008; Ríos Azuara, Pérez Flores, \& Ríos Alcolea, 2014)(Fernández Martínez, de la Cruz Márquez, Cueto Martín, Salazar Alonso, \& de la Cruz Campos, 2008; Ríos Azuara, Pérez Flores, \& RíosAlcolea, 2014).

De acuerdo con estudios realizados en universidades de México, Bélgica y Burundi (Adrien \& Onesphore, 2015; Flores Allende, Ruiz Juan, \& García Montes, 2009; Goossens et al., 2014; Adrien \& Onesphore, 2015) las personas interesadas en estudiar Educación Física o CIMOHU presentan un estilo de vida activo, y aunque en el buen sentido, poseen un mejor perfil de salud comparado con personas sedentarias, también son vulnerables a lesiones durante las sesiones de actividad física o deporte (González y Martínez, 2009). En el año 2012, la Coordinación de Vida Estudiantil de la Escuela de Educación Física y Deportes de la Universidad de Costa Rica, preguntó al estudiantado de primer ingreso «¿Por qué decidieron estudiar la carrera Ciencias del Movimiento Humano?». Todos los estudiantes manifestaron que eran personas a quienes les gustaba mucho realizar ejercicio físico, y el 63\% respondió que les gustaba mucho el deporte y que además lo practicaban (Escuela de Educación Física y Deportes, 2012b). Ese mismo año, pero con estudiantes de distintos niveles, se realizó un diagnóstico de autoreporte de lesiones a una muestra de 99 estudiantes, y se encontró que el $70 \%$ de ellos ya habían sufrido algún tipo de lesión, y el $45 \%$ se había lesionado durante ese semestre (Escuela de Educación Física y Deportes, 2012a). Las lesiones más frecuentes fueron las de tipo muscular, al igual que las reportadas en otro estudio (Goossens et al., 2014). Se detectó además que el $67 \%$ manifestó haber faltado a clases al menos una vez debido a alguna lesión y el 33\% indicó no haber faltado a lecciones por dicha situación, sin que esto significara ausencia de dolor (Escuela de Educación Física y Deportes, 2012a). Castro, Chacón, Zurita y Espejo (2016) reportan que quienes han sufrido lesiones y practican deporte o ejercicio físico regular, presentan una capacidad de resiliencia que aumenta en los niveles de competencia mayores,.

Considerando esta realidad se propone conocer el historial de lesiones del estudiantado de primer ingreso a la carrera Ciencias del Movimiento Humano y determinar el riesgo de lesión y la tasa de incidencia del futuro profesional en el movimiento humano cuya labor está directamente relacionada con la suma de horas de ejercicio.

\section{Metodología}

\section{Participantes}

La población del estudio fueron todos los estudiantes de primer ingreso de la carrera de CIMOHU de la Universidad de Costa Rica matriculados en el primer semestre del año 2014. El total de mujeres fue de 27 y de hombres $24(\mathrm{~N}=51)$.

\section{Instrumentos de medición}

Para describir las características antropométricas de los participantes, se midió la estatura corporal en cm, para lo cual se utilizó un estadiómetro metálico fijo (Novel Products Inc., modelo DES 290-337, Rockton, USA) con una precisión de $0.5 \mathrm{~cm}$. El peso corporal en kg se midió con una balanza electrónica marca Tanita ${ }^{\circledR}$ (modelo BF-350 W, Arlington Heights, IL, USA), con una precisión de 0.1 g. Con los valores de la estatura y el peso corporal se calculó el índice de masa corporal $(\mathrm{IMC}=\text { peso en kg/(talla en metros) })^{2}$ (American College of
Sports Medicine, 2014). Finalmente, la composición corporal (\% de masa grasa y masa magra corporal) se obtuvo por medio de absorciometría de rayos X de energía dual (DXA) marca General Electric ${ }^{\circledR}$ (modelo Lunar Prodigy Advance, Madison, WI, USA) con la versión de software enCore 2011, \# 13,60,033. Las valoraciones del DXA fueron realizadas por personal entrenado y certificado por el Ministerio de Salud Costaricense. El error de medición del DXA es $<2 \%$ (Carbuhn, Fernandez, Bragg, Green, \& Crouse, 2010; Valdimarsson, Alborg, Duppe, Nyquist, \& Karlsson, 2005).

Posteriormente se utilizó un cuestionario-diagnóstico diseñado por especialistas en CIMOHU y un profesional en medicina para la sección de semiología clínica en detección de lesión previa y aguda. El cuestionario constaba de información general del estudiante, antecedentes de lesiones previas, tratamientos recibidos, hábitos en la práctica de prevención de lesión y tiempo de exposición al ejercicio.

En este estudio, lesión deportiva se definió como «cualquier lesión que ocurre como resultado de la actividad deportiva y que causa una o más de las siguientes situaciones: el sujeto tiene que detener la actividad física, y/o no puede participar completamente en el siguiente entrenamiento, y/o no puede ir a trabajar, y/o necesita atención médica» (van Mechelen et al., 1996, p. 1172). También se tomó en consideración la cantidad de días de incapacidad que puede causar una lesión. Al respecto, aunque se han reportado diferentes clasificaciones, como por ejemplo, la de la National Athletic Injury/Illness Reporting System (NAIRS), la Injury Surveillance System (ISS) y laAthletic Injury Monitoring System (AIMS) (Zemper \& Dick, 2007), la definición que se utilizó en este estudio fue la de la NAIRS (Vinger, 1981), que clasifica las lesiones según la cantidad de días de incapacidad: «lesión menor» (1 a 7 días), «lesión moderada» (8 a 21 días), y «severa» (> 21 días o «daño permanente»).

\section{Procedimientos}

$\mathrm{Al}$ inicio del ciclo lectivo, se invitó a los estudiantes de primer ingreso a colaborar en el estudio. Primero, se les explicó el objetivo del estudio y voluntariamente decidieron participar todos los estudiantes, quienes completaron el consentimiento informado, el cual fue previamente aprobado por el Comité Ético Científico de la Universidad de Costa Rica. Posteriorme nte, completaron un cuestionario de antecedentes médicos y de actividad física. Este cuestionario contenía preguntas acerca del historial de lesiones, hábitos de realizar calentamiento, estiramiento y entrenamiento de fuerza muscular o potencia, y debían reportar, además, las horas de deporte o actividad física que realizaran extracurricularmente.

Una vez que el estudiante completaba la información, se les realizó un examen para evaluar variables de antropometría y composición corporal (i.e., DXA). Al iniciar las clases, como parte del protocolo del estudio, cada estudiante debía reportar por semana (lunes) si se lesionaba durante los 7 días anteriores. Aquel estudiante que se lesionara, procedía a sacar una cita con el médico del Centro de Investigación en Ciencias del Movimiento Humano para su respectiva revisión física y confirmación de la presencia de lesión y el tipo, así como la ubicación de la misma y su severidad. El profesional especialista en medicina, con más de 20 años de experiencia en la atención de lesiones relacionadas con la actividad física y el deporte, fue quien realizó la confirmación de las lesiones y sus características. En esta cita médica, se recopiló información acerca del día que ocurrió la lesión, si fue en clases de la carrera o en un contexto extracurricular, si fue haciendo deporte oficial o recreativo (i.e., ¿cómo se lesionó?), y además si la lesión era nueva o si se trataba de una lesión anterior que se volvía a presentar. Este protocolo se realizó durante todo el año lectivo 2014.

\section{Análisis estadístico}

Los análisis se realizaron con el IBM-SPSS Statistics, versión 20 (IBM Corporation, Armonk, New York). Se calculó la media y la desviación estándar $(\mathrm{M} \pm \mathrm{DE})$ para las variables medidas en escala continua. Se calcularon pruebas de Chi-cuadrado $\left(x^{2}\right)$ para determinar relaciones entrelas variables medidas en una escala categórica (Moncada- 
Jiménez, 2004, 2005b) e intervalos de confianza al 95\% (IC95\%). La significancia estadística se estableció a priori en $\mathrm{p}<0.05$.

Secalculó el riesgo de lesión y la tasa de incidencia de lesión mediante las fórmulas: a) Riesgo de lesión = nuevas lesiones/número de estudiantes; b) Tasa de incidencia de lesión = nuevas lesiones/horas participación por 1000 horas (Goossens et al., 2014).

\section{Resultados}

Se obtuvieron datos completos de 47 alumnos de primer ingreso; es decir, 92\% de la población inicial, pues 4 estudiantes (8\%) se retiraron por razones ajenas al estudio. Del total de participantes, 25 fueron mujeres (53.1\%) y 22 hombres (46.9\%). La edad promedio del grupo fue de $20.8 \pm 2.8$ años. En la Tabla 1 se describen las variables antropométricas por género.

El 48\% de la muestra de mujeres presentó valores de \% de grasa fuera de lo normal (9\% bajo peso y el 39\% sobrepeso). Por su parte, en los hombres, únicamente el 20\% presentó porcentaje de grasa alto y el resto fueron clasificados dentro del rango normal (American College Sports Medicine, 2014).

Al inicio del año escolar en marzo, ya el 31.8\% de los estudiantes de primer año presentaba lesiones. Las lesiones que más presentaron fueron los esguinces (10.6\% del total de los estudiantes), seguido por la inflamación a nivel de tendones o tendinitis (8.5\% del estudiantado). Como parte del historial de lesiones se documentó que el $45 \%$ de la población participante estuvo lesionada en los últimos 6 meses del año lectivo (Figura 1). De los alumnos lesionados que ingresaron con al menos una lesión, el $80 \%$ eran mujeres y el 20\% hombres $\left(x^{2}=27.5\right.$, $p$ $<0.001)$.

Riesgo de Lesión y Tasa de incidencia. Durante el año académico, se atendieron 28 estudiantes, 17 mujeres (60.7\%) y 11 hombres (39.3\%) $\left(x^{2}=1.57, \mathrm{p}<0.210\right)$. Se diagnosticaron 37 lesiones agudas nuevas, 24 en mujeres (64.9\%) y 13 en hombres (35.1\%). Para la población estudiada esto significa un riesgo total por lesión de 0.79 lesiones/estudiante/ año académico; en donde para las mujeres el riesgo de lesión fue de 0.96 y para los hombres de 0.59. En promedio, el tiempo de exposición al ejercicio del estudiantado fue de 13.14 horas/semana, durante 30 semanas de año lectivo. Esto corresponde a una tasa de incidencia de 2.00

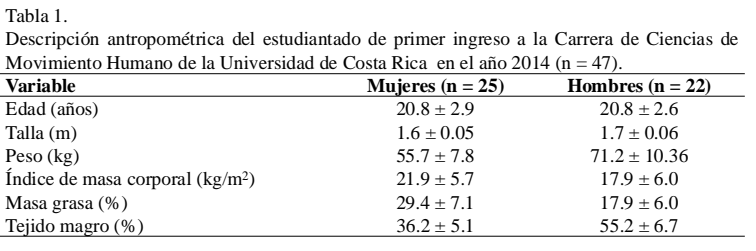

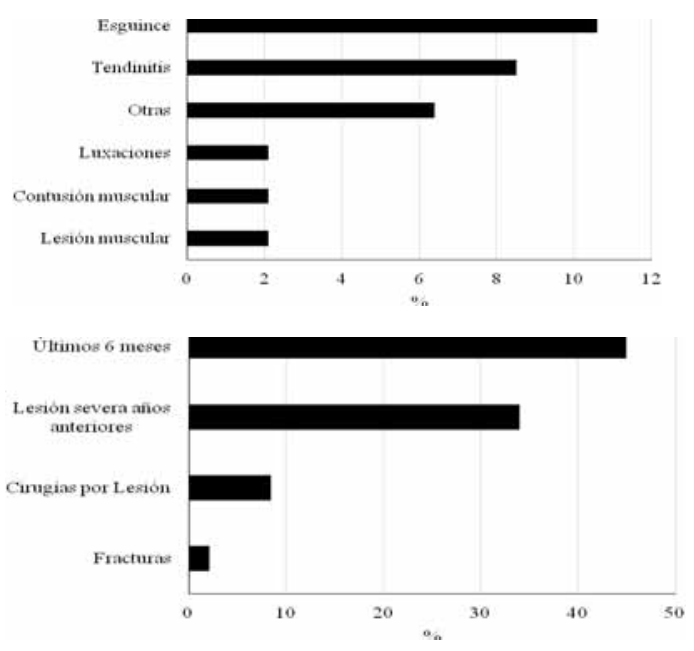

Figura 1. Tipo de lesión (panel superior) e historial de lesiones (panel inferior) al inicio del año Figura 1. Tipo de lesión (panel superior) e historial de lesiones (panel inferior) al inicio del
lectivo en estudiantes de primer año de la carrera en Ciencias del Movimiento Humano de la Universidad de Costa Rica.
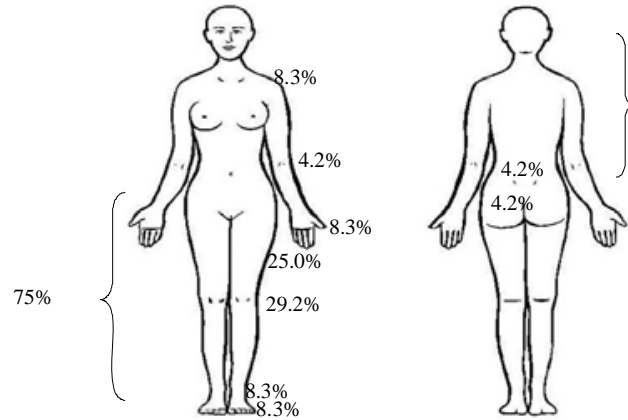

$25 \%$

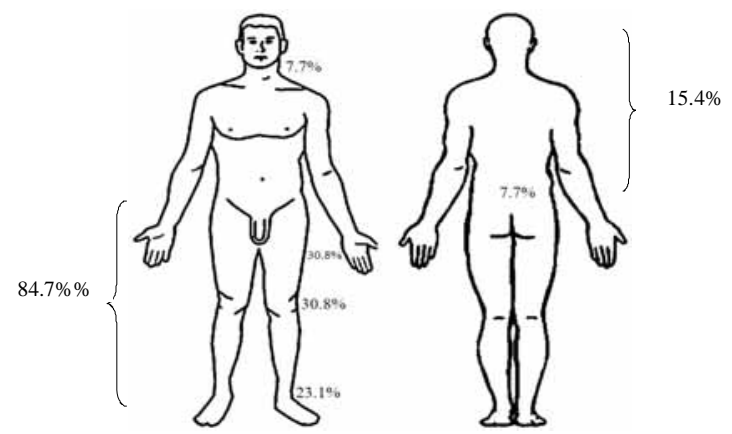

Figura 2. Porcentaje de lesiones en estudiantes de primer año de la carrera de Ciencias del Movimiento Humano de la Universidad de por zona anatómica. Panel superior muestra las mujeres y el panel inferior a los hombres.

lesiones/1000 horas de participación deportiva. Para las mujeres fue de $2.4(\mathrm{IC} 95 \%=1.64,3.62)$ y para hombres $1.50(\mathrm{IC} 95 \%=0.88,2.56)$ (Tabla 2).

Para el total de la población, el mes en el que se reportó el mayor porcentaje de lesiones fue setiembre (35.1\%). Para ambos géneros la mayoría de las lesiones ocurrieron en los miembros inferiores (mujeres $=75 \%$, hombres $=84.7 \%$ ). En las mujeres estas lesiones se ubicaron principalmente en el muslo (25\%) y la rodilla (29.2\%); y en los hombres se concentraron en el muslo (30.8\%), la rodilla (30.8\%) y el tobillo (23.1\%) (Figura 2).

Con respecto al contexto en que ocurrió la lesión, el 50\% de las mujeres se lesionaron en actividades intracurriculares (i.e., propias del plan de estudios), mientras que el $76.9 \%$ de los hombres se lesionaron principalmente en actividades extracurriculares (i.e., ajenas al plan de estudios). Con relación a la severidad de la lesión, según la clasificación de NAIRS (Vinger, 1981), se encontró que el 50\% de las lesiones en mujeres fueron de moderadas a severas y el otro $50 \%$ fueron severas.

Tabla 2.

Tiempo de exposición a lesiones en estudiantes de la carrera de Ciencias del Movimiento Humano de la Universidad en el año $2014(n=47)$.

\begin{tabular}{|c|c|c|c|c|c|c|c|}
\hline & \multicolumn{6}{|c|}{ Tiempo de exposición (h) } & \multirow[b]{2}{*}{$\begin{array}{c}\text { Riesgo } \\
\text { de lesión }\end{array}$} \\
\hline & \multicolumn{2}{|l|}{ Total } & \multicolumn{2}{|c|}{ Intracurricular } & \multicolumn{2}{|l|}{ Extracurricular } & \\
\hline Hombres $(n=22)$ & 8672.4 & & 5280.0 & & 3392.4 & & 0.59 \\
\hline Mujeres $(\mathrm{n}=25)$ & 9855.0 & & 6000.0 & & 3855.0 & & 0.96 \\
\hline \multirow{2}{*}{ Total $(n=47)$} & 18527.4 & & 11280.0 & & 7247.4 & & 0.79 \\
\hline & $\begin{array}{c}\text { Tasa } \\
\text { incidencia }\end{array}$ & IC95\% & $\begin{array}{c}\text { Tasa } \\
\text { incidencia }\end{array}$ & IC95\% & $\begin{array}{c}\text { Tasa } \\
\text { incidencia }\end{array}$ & IC95\% & \\
\hline Hombres & 1.50 & $0.88-2.56$ & 2.50 & $1.36-4.60$ & 3.11 & $1.78-5.43$ & \\
\hline Mujeres & 2.44 & $1.64-3.62$ & 3.11 & $1.78-5.43$ & 0.88 & $0.30-2.60$ & \\
\hline Total & 2.00 & $1.44-2.76$ & 1.95 & $1.29-2.96$ & 2.07 & $1.26-3.41$ & \\
\hline
\end{tabular}

Nota:

Riesgo de lesión = lesiones/estudiante/año escolar

Tasa de incidencia = lesiones $/ 1000 \mathrm{~h}$ de exposición IC95\% = Intervalo de confianza al 95\%

Tabla 3.

Asociación entre la frecuencia semanal de estiramiento o calentamiento y la presencia de lesiones. Los valores en las celdas representan frecuencias.

\begin{tabular}{|c|c|c|c|c|}
\hline \multirow[b]{2}{*}{ Frecuencia semanal } & \multicolumn{2}{|c|}{ Estiramiento } & \multicolumn{2}{|c|}{ Calentamiento } \\
\hline & Lesión & No Lesión & Lesión & No Lesión \\
\hline $\begin{array}{l}\text { Nunca } \\
\end{array}$ & 2 & 2 & 0 & 1 \\
\hline A veces & 1 & 4 & 5 & 5 \\
\hline Mayoría de veces & 8 & 11 & 5 & 9 \\
\hline Siempre & 5 & 14 & 6 & 16 \\
\hline Total & 16 & 31 & 16 & 31 \\
\hline
\end{tabular}


Sin embargo, solo el 35.2\% suspendió actividades físicas debido a la lesión. En el caso de los hombres, se encontró que el 75\% de las lesiones fueron de tipo menor y el $25 \%$ de moderadas a severas. Solamente $36.3 \%$ de los hombres suspendieron actividades físicas debido a la lesión.

Otro aspecto que se analizó, relacionado con la presencia de lesiones, fue el hábito de prácticas preventivas, como realizar estiramiento o calentamiento antes del ejercicio. Ninguna de esta prácticas se asoció significativamente con la presencia o ausencia de lesiones ( $p>0.05$ ). En la tabla 3, se detalla la frecuencia semanal con que la población estudiantil de primer año realizaba dichas prácticas; asimismo, los respectivos valores de la prueba de $x^{2}$ entre la población lesionada y la que no se lesionó según el tipo de práctica. Con respecto a la práctica de ejercicio contra resistencia para el fortalecimiento muscular tampoco se encontró una asociación significativa con la presencia de lesiones $\left(x^{2}=0.506, \mathrm{p}=\right.$ 0.477). Finalmente, en cuanto al historial de lesiones, no se encontró una asociación significativa entre los estudiantes que presentaron lesión en los últimos 6 meses y la aparición de nuevas lesiones $\left(x^{2}=3.116, \mathrm{p}=\right.$ $0.078)$.

\section{Discusión}

Atletas, deportistas y quienes dedican una alta cantidad de horas al ejercicio físico representan una población vulnerable para la presencia de lesiones, incluyendo poblaciones de niños hasta adultos mayores (Sundblad, Saartok, Engstrom, \& Renstrom, 2005). Como parte de esta población se encuentran también los estudiantes de Educación Física y Deportes o Ciencias del Movimiento Humano. Por ello, el propósito del estudio fue conocer la incidencia de lesiones y el riesgo de lesión en el estudiantado de primer año de la Carrera Ciencias del Movimiento Humano de la Universidad de Costa Rica durante el año lectivo 2014.

El riesgo de lesión encontrado en el presente estudio fue de 0.79 para 47 estudiantes de primer año de la Carrera de CIMOHU de la Universidad de Costa Rica. Al comparar este riesgo con el reportado en el estudio de Goosens et al. (2014), donde el riesgo de lesión reportado fue de 0.85 en 128 estudiantes de primer ingreso a la misma carrera, se puede concluir que son niveles de riesgo similares.

En cuanto a la ubicación de las nuevas lesiones, los resultados del presente estudio concuerdan con los del estudio de Goosens et al. (2014), quienes encontraron que es en el tren inferior donde se presentan la mayor cantidad de lesiones, tanto en hombres como en mujeres.

Con respecto a la tasa de incidencia se obtuvieron valores muy similares, de 2.00 lesiones/1000 horas de exposición para los estudiantes de CIMOHU y 1.91 lesiones/1000 horas de exposición para los estudiantes de Educación Física del estudio de Goosens et al. (2014). En cuanto a las horas de exposición al ejercicio, también se reportaron resultados similares en ambos estudios, ya que los estudiantes de CIMOHU se exponen al ejercicio un total de $13.14 \mathrm{~h} / \mathrm{semana}$ y en el estudio de Goosens et al. (2014), el tiempo de exposición fue de 15.41 h/semana.

En el presente estudio, una tercera parte de las lesiones sucedieron en el segundo semestre del año lectivo, específicamente en el mes de setiembre, cuando ya se había cursado más de un mes de clases del segundo semestre. Estas condiciones no concuerdan con la investigación realizada por Goossens et al. (2014), quienes reportaron lesiones en estudiantes de Educación Física durante los primeros meses de ingreso a clases.

Los resultados del presente estudio concuerdan con las características curriculares del primer año de carrera, ya que los estudiantes pasan de cursar un semestre de menor actividad física (i.e., menos cursos prácticos) a uno de mayor actividad física (i.e., más cursos de deportes). En el segundo semestre de este primer año, los estudiantes deben matricular el curso deportivo denominado «Pedagogía de la Gimnasia», el cual por su naturaleza demanda mayor exigencia física. De acuerdo con el estudio epidemiológico de Núñez, Gutierrez-Sánchezy Santana(2013), se determinó que quienes practican gimnasia aeróbica, o bien, competitiva, tienden a presentar lesiones a nivel muscular y en extremidades inferiores. Asimismo, Almeida, Williams, Shaffer, y Brodine (1999) y Barraza Gómez y Rodriguez Rodriguez (2011), consideran que al incrementarse de forma abrupta el volumen de trabajo o esfuerzo físico se aumenta el riesgo de lesiones. Lo que plantean estos investigadores es reflejo de las condiciones que se encontraron en el presente estudio.

En cuanto a la población lesionada, los resultados de esta investigación indican que el 59.5\% de los estudiantes de primer ingreso de la carrera de CIMOHU de la Universidad de Costa Rica presentaron una lesión nueva a lo largo del año académico de estudio. Estos datos son superiores a los previamente reportados en otra investigación con estudiantes de Educación Física en Bélgica (Goossens et al., 2014), en donde se encontró que alrededor del $50 \%$ de los estudiantes presentaron lesiones durante un año de seguimiento.

Con respecto a los hábitos de realizar actividades preventivas de lesiones, los estudiantes del presente estudio reportaron ejecutar estiramiento (91.5\%) y calentamiento (100\%), y poco menos del 50\% reportaron practicar ejercicio contra resistencia; sin embargo, estas estrategias no se asociaron con la presencia de lesiones. Es decir, realizar este tipo de actividades no les garantizólibrarse de lesiones. La literatura publicada respecto de estos temas no es contundente; en el caso de los calentamientos y estiramientos algunos han encontrado beneficios en la prevención de lesiones y otros no (Fradkin, Zazryn, \& Smoliga, 2010; Herman, Barton, Malliaras, \& Morrissey, 2012; McCrary, Ackermann, \& Halaki, 2015; Sampaio-Jorge et al., 2014).

Aunque para el presente estudio no se preguntó acerca del tipo de calentamiento o estiramiento específico que practicaban, sería importante atender algunos estudios recientes en los cuales se recomienda realizar ejercicios de propiocepción para prevenir las lesiones (Herman et al., 2012; Owoeye, Akinbo, Tella, \& Olawale, 2014). Por ejemplo, en Nigeria se llevó a cabo un estudio en el que compararon la propuesta de ejercicios propioceptivos denominado FIFA 11+ con la de calentamiento-estiramiento tradicional en fútbol (Owoeye et al., 2014). Los investigadores encontraron que el programa FIFA 11+ logró reducir en un $41 \%$ la presencia de lesiones en general y un $48 \%$ las lesiones en el tren inferior. Asimismo, otros estudios han comprobado el beneficio de este y otros programas de calentamiento neuromuscular en deportistas (Barengo et al., 2014), incluso en los de corta edad(Bien, 2011; Grooms, Palmer, Onate, Myer, \& Grindstaff, 2013); sin embargo, todavía hace falta investigar acerca de los programas de ejercicios de propiocepción con poblaciones de estudiantes de Educación Física y CIMOHU.

Con respecto al beneficio del entrenamiento contra-resistencia, se ha puesto a prueba especialmente el entrenamiento de tipo excéntrico para fortalecer los grupos musculares antagonistas en la fase de desaceleración de acciones concéntricas de alta intensidad, ya que es en éstos donde se presentan principalmente las lesiones. En un estudio realizado en Australia (Clark, Bryant, Culgan, \& Hartley, 2005), se analizó el efecto de este tipo de entrenamiento contra-resistencia y se hallaron adaptaciones neuromusculares favorables en la prevención de lesiones. Así, otros estudios, también apoyan el beneficio del entrenamiento contra-resistencia de este tipo en la prevención de lesiones (de Hoyo et al., 2015; Faigenbaum \& Myer, 2010; Petersen, Thorborg, Nielsen, Budtz-Jorgensen, \& Holmich, 2011).

Otro aspecto importante del presente estudio, es el contexto en el que principalmente ocurren las lesiones. Para el caso de las mujeres éstas se lesionan especialmente durante los cursos lectivos, lo que concuerda con el estudio de Goossens et al. (2014), mientras que, en el caso de los hombres, es en actividades extracurriculares tales como sesiones de entrenamiento, en juegos oficiales, las clases de gimnasio y otros espacios recreativo-deportivos indicados por los estudiantes.

En conclusión, un importante porcentaje de estudiantes (31.8\%) ingresa a la carrera con alguna lesión y el 80\% de esta población, fueron mujeres. El 59.5\% del estudiantado de la carrera de CIMOHU se lesiona durante su primer año de estudios, con mayor incidencia en el segundo semestre del año lectivo, y las lesiones principales tanto en mujeres como hombres, se presentaron en rodilla y músculos del tren inferior. Se determinó, que las prácticas de estiramiento, calentamiento y entrenamiento contra resistencia no se asociaron significativamente con la pre- 
sencia de lesiones; sin embargo, este tema de actividades protectoras requiere de mayor investigación. Se recomienda realizar estudios longitudinales que permitan dar seguimiento al estudiantado durante todo el plan de estudios y conocer así la evolución de las lesiones en esta población. Por otro lado, es importante fortalecer los programas educativos y preventivos desde que el estudiante ingresa a la carrera.

\section{Agradecimiento}

Se agradece el apoyo profesional al profesor e investigador Dr. José Moncada Jiménez, Director del Centro de Investigación en Ciencias del Movimiento Humano.

\section{Referencias}

Adrien, N., \& Onesphore, H. (2015). Lesions and injuries associated to sport and physical education in Burundi elementary schools. International Journal of Sports Sciences \& Fitness, 5(2), 10-10.

Almeida, S. A., Williams, K. M., Shaffer, R. A., \& Brodine, S. K. (1999). Epidemiological patterns of musculoskeletal injuries and physical training. Med Sci Sports Exerc, 31(8), 1176-1182.

American College of Sports Medicine. (2014). ACSM's Guidelines for Exercise Testing and Prescription ( $9^{\text {th }}$ ed.). Philadelphia, PA: Wolters Kluwer, Lippincott Williams \& Wilkins.

Barengo, N. C., Meneses-Echavez, J. F., Ramirez-Velez, R., Cohen, D. D., Tovar, G., \& Bautista, J. E. (2014). The impact of the FIFA11+ training program on injury prevention in football players: a systematic review. Int J Environ Res Public Health, 11(11), 11986-12000. doi:10.3390/ijerph111111986

Barraza Gómez, F., \& Rodriguez Rodriguez, F. (2011). Comparación de la masa muscular y masa grasa de estudiantes de primer año de educación física. Motricidad Humana, 12(1), 34-39.

Bien, D. P. (2011). Rationale and implementation of anterior cruciate ligament injury prevention warm-up programs in female athletes. J Strength Cond Res, 25(1), 271-285. doi:10.1519/JSC.0b013e3181fb4a5a

Carbuhn, A. F., Fernandez, T. E., Bragg,A. F., Green, J. S., \& Crouse, S. F. (2010) Sport and training influence bone and body composition in women collegiate athletes. J Strength Cond Res, 24(7), 1710-1717. doi:10.1519/ JSC.0b013e3181d09eb3

Castro Sanchez, M., Chacón Cuberos, R., Zurita Ortega, F., \& Espejo Garcés, T. (2016). Levels of resilience based on sport discipline, competitive level and sport injuries. RETOS-Nuevas Tendencias en Educación Física, Deporte y Recreación, (29), 162-165.

Clark, R., Bryant, A., Culgan, J.-P., \& Hartley, B. (2005). The effects of eccentric hamstring strength training on dynamic jumping performance and isokinetic strength parameters: a pilot study on the implications for the prevention of hamstring injuries. Physical Therapy in Sport, 6(2), 67-73. doi:http://dx.doi.org 10.1016/j.ptsp.2005.02.003

de Hoyo, M., Pozzo, M., Sanudo, B., Carrasco, L., Gonzalo-Skok, O., DominguezCobo, S., \& Moran-Camacho, E. (2015). Effects of a 10-week in-season eccentric-overload training program on muscle-injury prevention and performance in junior elite soccer players. Int J Sports Physiol Perform, 10(1), 46-52. doi:10.1123/ijspp.2013-0547

Escuela de Educación Física y Deportes. (2012a). Cuestionario de Lesiones a estudiantes de la Carrera Ciencias del Movimiento Humano. Universidad de Costa Rica. San José, Costa Rica.

Escuela de Educación Física y Deportes. (2012b). Encuesta a estudiantes de primer ingreso a la carrera de Ciencias del Movimiento Humano. Universidad de Costa Rica. San José, Costa Rica.

Faigenbaum, A. D., \& Myer, G. D. (2010). Resistance training among young athletes: safety, efficacy and injury prevention effects. Br JSports Med,44(1), 5663. doi:10.1136/bjsm.2009.068098

Fernández Martínez, A., de la Cruz Márquez, J. C., Cueto Martín, B., Salazar Alonso, S., \& de la Cruz Campos, J. C. (2008). Predicción de lesiones deportivas mediante modelos matemáticos. Apunts. Medicina de L'Esport, $157,41-44$

Fiuza-Luces, C., Garatachea, N., Berger, N. A., \& Lucia, A. (2013). Exercise is the real polypill. Physiology (Bethesda), 28(5), 330-358. doi:10.1152 physiol.00019.2013

Flores Allende, G, Ruiz Juan, F., \& García Montes, M. E. (2009). Relación de algunos correlatos biológicos y demográficos con lapráctica físico-deportiva en estudiantes universitarios: el caso de la Universidad de Guadalajara, México. RICYDE. Revista Internacional de Ciencias del Deporte, 5(14), 59-80.

Fradkin, A. J., Zazryn, T. R., \& Smoliga, J. M. (2010). Effects of warming-up on physical performance: a systematic review with meta-analysis. JStrength Cond
Res, 24(1), 140-148. doi:10.1519/JSC.0b013e3181c643a0

Goossens, L., Verrelst, R., Cardon, G, \& De Clercq, D. (2014). Sports injuries in physical education teacher education students. Scand J Med Sci Sports, 24(4), 683-691. doi:10.1111/sms.12054

Grooms, D. R., Palmer, T., Onate, J. A., Myer, G. D., \& Grindstaff, T. (2013). Soccer-specific warm-up and lower extremity injury rates in collegiate male soccer players. J Athl Train, 48(6), 782-789. doi:10.4085/1062-6050-48.4.08

Herman, K., Barton, C., Malliaras, P., \& Morrissey, D. (2012). The effectiveness of neuromuscular warm-up strategies, that require no additional equipment, for preventing lower limb injuries during sports participation: a systematic review. BMCMed, 10, 75. doi:10.1186/1741-7015-10-75

McCrary, J. M.,Ackermann, B. J., \& Halaki, M. (2015). A systematic review of the effects of upper body warm-up on performance and injury. Br J Sports Med, 49(14), 935-942. doi:10.1136/bjsports-2014-094228

Meneses, M., \& Moncada, J. (2004). Hábitos de exposición solar y conocimientos sobre el cuidado de la piel en educadores físicos mexicanos, hondureños y costarricenses. Revista Costarricense de Salud Pública, 13(25), 34-41.

Moncada-Jiménez, J. (2004). Métodos estadísticos utilizados en las ciencias del movimiento humano. Revista Educación, 28(2), 279-287.

Moncada-Jiménez, J.(2005a). 2005:Año Internacional del Deporte y la Educación Física. Revista Educación, 29(2), 233-247.

Moncada-Jiménez, J. (2005b). Estadística para las ciencias del movimiento humano. San José, Costa Rica: Editorial de la Universidad de Costa Rica.

Moncada-Jiménez, J., Meneses-Montero, M., Hidalgo-Matlock, B., \& GranadosChavarría, C. (2004). Lesiones cutáneas en educadores físicos costarricenses. Acta Médica Costarricense, 46(3), 132-138.

Núñez, R. A., Gutierrez-Sánchez, A. G., \& Santana, M. V.(2013). Las lesiones en la gimnasia aeróbica: artículo de revisión. Revista Internacional de Medicinay Ciencias de la Actividad Física y del Deporte, (49), 12-16.

Owoeye, O. B., Akinbo, S. R., Tella, B.A., \& Olawale, O.A.(2014). Efficacy of the FIFA11+ Warm-Up Programmein MaleYouth Football:ACluster Randomised Controlled Trial. J Sports Sci Med, 13(2), 321-328.

Petersen, J., Thorborg, K., Nielsen, M. B., Budtz-Jorgensen, E., \& Holmich, P. (2011). Preventive effect of eccentric training on acute hamstring injuries in men's soccer: a cluster-randomized controlled trial. Am J Sports Med,39(11), 2296-2303. doi:10.1177/0363546511419277

Ríos Azuara, D., Pérez Flores, D., \& RíosAlcolea, M. (2014). Epidemiología de las lesiones deportivas en países de la unión europea [Epidemiology of sports injuries in european union countries]. Revista Internacional de Medicina y Ciencias de la Actividad Física y el Deporte, 14(55), 479-494.

Sampaio-Jorge, F., Rangel, L. F. C., Mota, H. R., Morales, A. P., Costa, L., Coelho, G. M., \& Ribeiro, B. G (2014). Acute Effects of Passive Stretching on Muscle Power Performance. Journal of Exercise Physiology Online, 17(6), 8189.

Sundblad, G., Saartok, T., Engstrom, L. M., \& Renstrom, P. (2005). Injuries during physical activity in school children. Scand J Med Sci Sports, 15(5), 313323. doi:10.1111/j.1600-0838.2004.00419.x

Twellaar, M., Verstappen, F. T., \& Huson,A. (1996). Is prevention of sports injuries a realistic goal? A four-year prospective investigation of sports injuries among physical education students. Am J Sports Med, 24(4), 528-534.

Valdimarsson, O., Alborg, H. G., Duppe, H., Nyquist, F., \& Karlsson, M. (2005) Reduced training is associated with increased loss of BMD. JBone Miner Res, 20(6), 906-912. doi:10.1359/jbmr.050107

van Mechelen, W., Hlobil, H., \& Kemper, H. C. (1992). Incidence, severity, aetiology and prevention of sports injuries. A review of concepts. Sports Med, 14(2), 82-99.

van Mechelen, W., Twisk, J., Molendijk, A., Blom, B., Snel, J., \& Kemper, H. C. (1996). Subject-related risk factors for sports injuries: a 1-yr prospective study in young adults. Med Sci Sports Exerc, 28(9), 1171-1179.

Vinger, P. F.(1981). Ocular sports injuries. Principles of protection. Int Ophthalmol Clin, 21(4), 149-161.

Zemper, E. D., \& Dick, R. W. (Eds.). (2007). Epidemiology of athletic injuries (2n ed.). Philadelphia, PA: Wolkers Kluver, Lippincott Williams \& Wilkins.

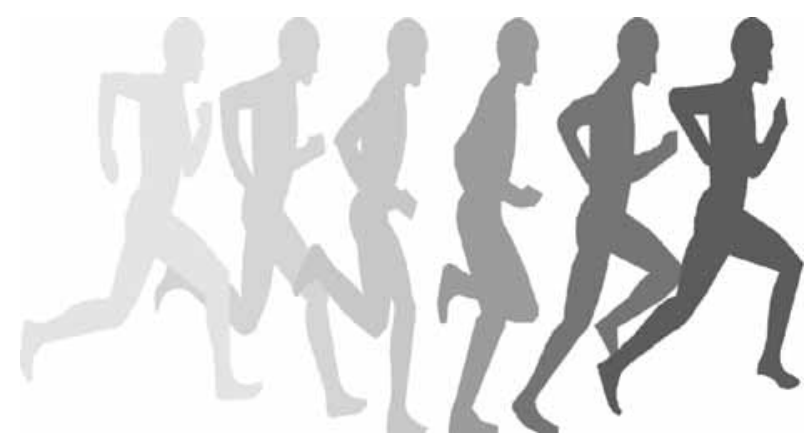

